\title{
NONLINEAR STOCHASTIC FUNCTIONAL INTEGRAL EQUATIONS IN THE PLANE
}

\author{
JAN TURO \\ Technical University of Gdańsk \\ Department of Mathematics \\ 80-952 Gdańsk, Narutowicza 11/12 Poland
}

(Received April, 1995; Revised August, 1995)

\begin{abstract}
Local or global existence and uniqueness theorems for nonlinear stochastic functional integral equations are proved. The proofs are based on the successive approximations methods. The formulation includes retarded arguments and hereditary Volterra terms.
\end{abstract}

Key words: Stochastic Equations, Two-Parameter Wiener Process, Successive Approximations.

AMS (MOS) subject classifications: $60 \mathrm{H} 20$.

\section{Introduction}

In this paper we shall consider a stochastic functional integral equation of the following type:

$$
\begin{gathered}
u(z)=\psi(z)=\int_{I_{z}} f\left(\tau, u_{\tau}\right) d \tau+\int_{I_{z}} g\left(\tau, u_{\tau}\right) d w(\tau), \quad z=(x, y) \in \mathbb{R}_{+}^{2} \\
u(z)=\varphi(z), \quad z \in I_{0} .
\end{gathered}
$$

The second integral in equation (1) is a stochastic integral in the plane [1], [7].

Equations of this type appear when one rewrites the partial stochastic functional differential equation

$$
\begin{gathered}
\frac{\partial^{2} u}{\partial x \partial y}(x, y)=f\left(x, y, u_{(x, y)}\right)+g\left(x, y, u_{(x, y)}\right) \frac{\partial^{2} w}{\partial x \partial y}(x, y), \quad(x, y) \in \mathbb{R}_{+}^{2} \\
u(x, y)=\varphi(x, y), \quad(x, y) \in I_{0}
\end{gathered}
$$

where $\frac{\partial^{2} w}{\partial x \partial y}$ is a space time white noise, as an integral equation.

An existence and uniqueness result for equation (3) (with $h=0$ ) when the drift coefficient $f$ and the diffusion coefficient $g$ satisfy a Lipschitz condition have been established by Gikhman and Pjasetskaja [2] and by Tsarenko [8]. In this paper, using the usual Picard iterative method, we investigate the local and global existence as well as uniqueness of solutions of problem (1), (2), without assuming a Lipschitz condition in the last argument for $f$ and $g$.

Partial differential equations with a retarded argument and differential-integral equations 
can be obtained from (1) by specializing given functions. Functional partial differential equations with operators of the Volterra type are also the special cases of (1) (see Section 6).

\section{Preliminaries}

Let us introduce a notation for rectangles. Suppose $z=(x, y)$ and $z^{\prime}=\left(x^{\prime}, y^{\prime}\right)$. We say that $z \leq z^{\prime}$ iff $x \leq x^{\prime}$ and $y \leq y^{\prime}$. We also write $z<z^{\prime}$ iff $x<x^{\prime}$ and $y<y^{\prime}$. We shall use the notation $I_{z, z^{\prime}}$ for the closed rectangle $\left[x, x^{\prime}\right] \times\left[y, y^{\prime}\right]$. We denote the rectangle $I_{0, z}=[0, x] \times[0, y]$ by $I_{z}$. The parameter space will be the square of the plane $I_{a}$, where $a=\left(a_{1}, a_{2}\right)$.

Let $J=I_{-h, 0}$, where $h=\left(h_{1}, h_{2}\right)$ and $h_{1}, h_{2}>0$. By $C=C(J, R)$ we denote the Banach space of all continuous functions $u: J \rightarrow \mathbb{R}$ endowed with supremum norm $\|u\|=\sup _{\xi \in J}|u(\xi)|$. Let $\tilde{I}=I_{-h, a}$ and $I_{0}=\overline{\widetilde{I} \backslash I_{a}}$. For any function $u: \tilde{I} \rightarrow \mathbb{R}$ and a fixed $z \in I_{a}$ we define function $u_{z}: J \rightarrow \mathbb{R}$ by $u_{z}(\xi)=u(z+\xi)$ for $\xi \in J$.

Let $(\Omega, \mathscr{F}, \mathscr{P})$ be a complete probability space. By $w$ we denote a two-parameter Wiener process. We introduce a family $\mathcal{F}_{z}, z \in I$, of $\sigma$-algebras of subsets of $\Omega$ with the following properties:

(i) $\quad \mathscr{F}_{z} \subset \mathcal{F}_{z^{\prime}}$ for $z \leq z^{\prime} \leq a$;

(ii) for every $z \in I_{a}, w(z)$ is $\mathscr{F}_{z}$-measurable;

(iii) for every $z \in I_{a}$, the Wiener measure of the rectangle $I_{z, z+\lambda} \subset I_{a}$, which can be introduced as $w(z+\lambda)-w\left(z+\lambda_{1}^{0}\right)-w\left(z+\lambda_{2}^{0}\right)+w(z)$ is independent of $\mathcal{F}_{z+\lambda_{1}^{0}}$ and $\mathcal{F}_{z+\lambda_{2}^{0}}$ for $\lambda=\left(\lambda_{1}, \lambda_{2}\right) \in \mathbb{R}_{+}^{2}$, where $\lambda_{1}^{0}=\left(\lambda_{1}, 0\right), \lambda_{2}^{0}=\left(0, \lambda_{2}\right), \mathbb{R}_{+}=[0, \infty)$.

Let $\mathscr{B}(\varphi)$ be the set of all functions $v: I_{a} \times \Omega \rightarrow \mathbb{R}$ with the following conditions:

(a) $\quad v(z, \cdot): \Omega \rightarrow \mathbb{R}$ is measurable for each fixed $z \in I_{a}$;

(b) $\quad v(\cdot, \omega): I \rightarrow \mathbb{R}$ is continuous for a.e. fixed $\omega \in \Omega$;

(c) $v(z, \omega)=\varphi(z, \omega)$ for $z \in I_{0}, \omega \in \Omega$.

It is easy to prove, similarly like in [5], by Borel-Cantelli lemma that $\mathscr{B}(\varphi)$ with the norm $\|v\|_{\mathscr{B}(\varphi)}=\left(E\left(\sup _{z \in \tilde{I}}|v(z, \omega)|^{2}\right)^{1 / 2}\right.$ is a Banach space.

Let $L^{2}(\Omega, C)$ be the set of all $C(J, R)$-valued, mean square integrable functions on $\Omega$ and let $S$ be the closed ball of center $\gamma$ with radius $r$ in $L^{2}(\Omega, C)$, that is, $S=S(\gamma, r)=\left\{v \in L^{2}(\Omega, C)\right.$ : $\left.E\|v-\gamma\|^{2} \leq r\right\}$, where $r>0$ and

$$
\gamma(z)= \begin{cases}\psi(z), & z \in I_{a} \\ \varphi(z), & z \in I_{0}\end{cases}
$$

\section{Assumptions and Lemmas}

\section{Assumption $\boldsymbol{H}_{\mathbf{1}}$ : Suppose that}

$1^{0}$ the functions $f, g: I_{a} \times C \rightarrow \mathbb{R}$ are measurable and $f(z, \cdot), g(z, \cdot)$ are continuous for each fixed $z \in I_{a}$;

$2^{0} \quad$ there is a function $H: \mathbb{R}_{+}^{3} \rightarrow \mathbb{R}_{+}, H(\cdot, v)$ is locally integrable for each fixed $v \in \mathbb{R}_{+}$and $H(z, \cdot)$ is continuous, monotone nondecreasing for each fixed $z \in \mathbb{R}_{+}^{2}$ such that

$$
E|f(z, v)|^{2}+E|g(z, v)|^{2} \leq H\left(z, E\|v\|^{2}\right),
$$


for all $z \in I_{a}$ and all $v \in S$;

$3^{0} \quad \gamma(\cdot, \omega): \tilde{I} \rightarrow \mathbb{R}$ is continuous for a.e. $\omega \in \Omega, \gamma(z, \cdot)$ is $\mathcal{F}_{0}$-measurable, independent of the two parameter Wiener process $w$ and $\gamma^{*}=E\left(\sup _{z \in \tilde{I}}|\gamma(z)|^{2}\right)<\infty$.

Now, we define the sequence of successive approximations $\left\{u^{n}(z)\right\}$ as follows:

$$
u^{n+1}(z)=\left\{\begin{array}{cc}
\psi(z)+\int_{I_{z}} f\left(\tau, u_{\tau}^{n}\right) d \tau+\int_{I_{z}} g\left(\tau, u_{\tau}^{n}\right) d w(\tau), & z \in I_{a} \\
\varphi(z), & z \in I_{0}, \\
u^{0}(z)=\gamma(z), \quad z \in \tilde{I} . &
\end{array}\right.
$$

Lemma 1: Let Assumption $H_{1}$ be satisfied. Then, there is $\bar{a}=\left(\bar{a}_{1}, \bar{a}_{2}\right)$ such that $0<\bar{a}<a$ and the sequence of the functions $\left\{E\left\|u_{z}^{n}\right\|^{2}\right\}, z \in I_{\bar{a}}$ is uniformly bounded.

Proof: It is easy to show that the integrals on the right-hand side of (5) are well defined. Now, let us note, by condition $2^{0}$ of $H_{1}$, that the problem

$$
\begin{aligned}
& D_{z} v(z)=3\left(4+a_{1} a_{2}\right) H(z, v(z)) \\
& v(x, 0)=\alpha(x), \quad v(0, y)=\beta(y)
\end{aligned}
$$

has a local solution with any initial functions $\alpha$ and $\beta, \alpha(0)=\beta(0)$ [3], [4], where $D_{z}=\frac{\partial^{2}}{\partial x \partial y}$. Take $\alpha$ and $\beta$ such that $\alpha(x)+\beta(y)-\beta(0)>3 \gamma^{*},(x, y) \in I_{a}$, and let $v$ be the local solution of (5) with the initial functions $\alpha$ and $\beta$. Now, we shall show that

$$
E\left\|u_{z}^{n}\right\|^{2}<v(z)
$$

and

$$
\left\|u_{z}^{n}-\gamma_{z}\right\|^{2} \leq r
$$

hold for $z \in I_{\bar{a}}, n=1,2, \ldots$

Utilizing Doob's and Schwarz's inequalities and condition (4) we obtain $a_{0}$ such that $0<a_{0}<a$ and

$$
\begin{gathered}
E\left\|u_{z}^{1}\right\|^{2}=\sup _{\xi \in J}\left|u^{1}(z+\xi)\right|^{2} \\
\leq 3 E\left(\sup _{\xi \in J}|\gamma(z+\xi)|^{2}+\sup _{\theta \in I_{\eta, z}}\left|\int_{I_{\theta}} f\left(\tau, u_{\tau}^{0}\right) d \tau\right|^{2}+\sup _{\theta \in I_{\eta, z}}\left|\int_{I_{\theta}} g\left(\tau, u_{\tau}^{0}\right) d w(\tau)\right|^{2}\right) \\
\leq 3 \gamma^{*}+3\left(4+a_{1} a_{2}\right) \int_{I_{z}} H\left(\tau, E\left\|u_{\tau}^{0}\right\|^{2}\right) d \tau<v(t),
\end{gathered}
$$

for all $z \in I_{a}$, since $v(z) \geq \alpha(x)+\beta(y)-\beta(0)>3 \gamma^{*}$ and $v(z)$ is the local solution of (6), where $\eta=\left(\max \left(0, x-h_{1}\right), \max \left(0, y-h_{2}\right)\right)$.

Since the function $H(z, v(z))$ is integrable on $I_{a_{0}}$, there is $\bar{a}$ such that $0<\bar{a}<a_{0}$ and

$$
E\left\|u_{z}^{1}-\gamma_{z}\right\|^{2} \leq 2 E\left(\sup _{\theta \in I_{z}}\left|\int_{I_{\theta}} f\left(\tau, u_{\tau}^{0}\right) d \tau\right|^{2}\right)+2 E\left(\sup _{\theta \in I_{z}}\left|\int_{I_{\theta}} g\left(\tau, u_{\tau}^{0}\right) d w(\tau)\right|^{2}\right)
$$




$$
\leq 2\left(4+a_{1} a_{2}\right) \int_{I_{z}} H(\tau, v(\tau)) d \tau \leq r
$$

for all $z \in I_{\bar{a}}$. Assume that (8) and (9) hold for $n=k$. Then, we have for $n=k+1$,

$$
E\left\|u_{z}^{k+1}\right\|^{2} \leq 3 \gamma^{*}+3\left(4+a_{1} a_{2}\right) \int_{I_{z}} H\left(\tau, E\left\|u_{\tau}^{k}\right\|^{2}\right) d \tau<v(z)
$$

for all $z \in I_{\bar{a}}$. Next, utilizing Doob's and Schwarz's inequalities we get

$$
\begin{gathered}
E\left\|u_{z}^{k+1}-\gamma_{z}\right\|^{2} \leq 2\left(4+a_{1} a_{2}\right) \int_{I_{z}} H\left(\tau, E\left\|u_{\tau}^{k}\right\|^{2}\right) d \tau \\
\leq 2\left(4+a_{1} a_{2}\right) \int_{I_{z}} H(\tau, v(\tau)) d \tau \leq r
\end{gathered}
$$

for all $z \in I_{\bar{a}}$. Thus, by induction, (8) and (9) hold for all $n$. Since $v(z)$ is continuous on $I_{\bar{a}}$, there exists a real number $C>0$ such that $E\left\|u_{z}^{n}\right\|^{2}<C$ for all $z \in I_{\bar{a}}$ and every integer $n \geq 0$, which completes the proof of the lemma.

Assumption $\mathrm{H}_{2}$ : Suppose that

$1^{0}$ there is a function $G: I_{a} \times[0,4 r] \rightarrow \mathbb{R}_{+}, G(\cdot, v)$ is locally integrable for each fixed $v \in[0,4 r]$ and $G(z, \cdot)$ is monotone nondecreasing, continuous for each fixed $z \in I_{a}$ such that $G(z, 0)=0$ and

$$
E|f(z, v)-f(z, \bar{v})|^{2}+E|g(z, v)-g(z, \bar{v})|^{2} \leq G\left(z, E\|v-\bar{v}\|^{2}\right),
$$

for all $z \in I_{a}$ and all $v, \bar{v} \in S$;

$2^{0} \quad$ if a nonnegative continuous function $\eta$ satisfies the following inequality

$$
\eta(z) \leq A \int_{I_{z}} G(\tau, \eta(\tau)) d \tau
$$

for all $z \in I_{\bar{a}}$, where $A=2\left(4+a_{1} a_{2}\right)$ and $0<\bar{a} \leq a$ and if $\eta(0)=0$, then $\eta(z) \equiv 0$ on $I_{\bar{a}}$.

Similarly, as in [6] we can prove the following lemma containing sufficient conditions under which condition $2^{0}$ of $\mathrm{H}_{2}$ is satisfied.

Lemma 2: Assume that

$1^{0} \quad R(\cdot, v): \mathbb{R}_{+}^{2} \rightarrow \mathbb{R}_{+}$is locally integrable for each fixed $v \in \mathbb{R}_{+} ;$

$2^{0} \quad R(z, \cdot): \mathbb{R}_{+}^{+} \rightarrow \mathbb{R}_{+}^{+}$is continuous, monotone nondecreasing for each fixed $z \in \mathbb{R}_{+}^{2}$, and $R(z, 0) \equiv 0$ for all $z \in \mathbb{R}_{+}^{2}$;

$3^{0} \quad \eta(z)$ is a solution of the differential equation $D_{z} \eta(z)=R(z, \eta(z))$;

$4^{0} \quad$ if $\eta(\bar{z})=0$ for some $\bar{z} \in I_{a}$, then $\eta(z) \equiv 0$ for all $z \in I_{\bar{z}, a}$.

Then, if a nonnegative continuous function $\eta(z), z \in \mathbb{R}_{+}^{2}$ satisfies $\eta(0)=0$ and

for all $z \in I_{a}$, then $\eta(z) \equiv 0$ for all $z \in I_{a}$.

$$
\eta(z) \leq \int_{I_{z}} R(\tau, \eta(\tau)) d \tau
$$

Remark: As an example of a function which satisfies $2^{0}$ of $H_{2}$ we can take $R(z, v)=$ 
$\lambda(z) \mu(v), z \in \mathbb{R}_{+}^{2}, v \in \mathbb{R}_{+}$, where $\lambda: \mathbb{R}_{+}^{2} \rightarrow \mathbb{R}_{+}$is locally integrable and $\mu: \mathbb{R}_{+} \rightarrow \mathbb{R}_{+}$is a continuous, monotone nondecreasing function with $\mu(0)=0$ such that $\int_{0_{+}} 1 / \mu(v) d v=\infty$.

If $\mu(v)=v, v \in \mathbb{R}_{+}$and $\lambda(z)=L(L>0)$, then condition (10) implies global Lipschitz condition [2], [8].

\section{The Local Existence of Solutions}

Theorem 1: Let Assumptions $H_{1}$ and $H_{2}$ be satisfied. Then, the sequence $\left\{u^{n}(z)\right\}$ defined by (5) converges uniformly on some rectangle $I_{\bar{a}}$ to a unique local solution of (1), (2).

Proof: Let $I_{\bar{a}}$ be the rectangle which is obtained in Lemma 1. Now, we define a sequence $\left\{\delta_{k}\right\}$ of functions $\delta_{k}: I_{\bar{a}} \rightarrow \mathbb{R}_{+}$by

$$
\delta_{k}(z)=\sup \left\{\delta_{m n}(z): m \geq n \geq k\right\}
$$

where

$$
\delta_{m n}(z)=E\left\|u_{z}^{m}-u_{z}^{n}\right\|^{2}
$$

Since the sequence $\left\{E\left\|u_{z}^{n}\right\|^{2}\right\}, z \in I_{\bar{a}}$ is uniformly bounded by Lemma 1, we obtain a positive and real number $M$ such that

$$
\delta_{m n}(z) \leq 2 E\left(\left\|u_{z}^{m}\right\|^{2}+\left\|u_{z}^{n}\right\|^{2}\right)<M
$$

for all $z \in I_{\bar{a}}$. Next, we have that

$$
\begin{aligned}
& \left|\delta_{m n}(z)-\delta_{m n}(s)\right| \leq E\left(\left\|u_{z}^{m}-u_{z}^{n}\right\|+\left\|u_{s}^{m}-u_{s}^{n}\right\|\right)\left(\left\|u_{z}^{m}-u_{s}^{m}\right\|+\left\|u_{z}^{n}-u_{s}^{n}\right\|\right) \\
& \leq\left[E\left(\left\|u_{z}^{m}-u_{z}^{n}\right\|+\left\|u_{s}^{m}-u_{s}^{n}\right\|\right)^{2}\right]^{1 / 2} \cdot\left[E\left(2\left\|u_{z}^{m}-u_{s}^{m}\right\|^{2}+2\left\|u_{z}^{n}-u_{s}^{n}\right\|^{2}\right)\right]^{1 / 2}
\end{aligned}
$$

On the other hand, utilizing Doob's martingale inequality and Schwartz's inequality we get

$$
\begin{gathered}
E\left\|u_{z}^{n}-u_{s}^{n}\right\|^{2} \leq 2 a_{1} a_{2} E\left(\int_{I_{s, z}}\left|f\left(\tau, u_{\tau}^{n-1}\right)\right|^{2} d \tau\right)+8 E\left(\int_{I_{s, z}}\left|g\left(\tau, u_{\tau}^{n-1}\right)\right|^{2} d \tau\right) \\
\leq 2\left(4+a_{1} a_{2}\right) \int_{I_{s, z}} H\left(\tau, E\left\|u_{\tau}^{n-1}\right\|^{2}\right) d \tau \\
\leq 2\left(4+a_{1} a_{2}\right)|Q(z)-Q(s)|
\end{gathered}
$$

for all $z, s \in I_{\bar{a}}$ and all integers $n \geq 1$, where $Q(z)=\int_{I_{z}} H(\tau, v(\tau)) d \tau$, and $v(z)$ is a local solution of (6). Thus, we obtain a positive and real number $P$ such that

$$
\left|\delta_{m n}(z)-\delta_{m n}(s)\right| \leq P|Q(z)-Q(s)|^{1 / 2}
$$

for all $m \geq n \geq 0$ and $z, s \in I_{\bar{a}}$. Hence, we have

$$
0 \leq \delta_{k}(z)<M
$$


and

$$
\left|\delta_{k}(z)-\delta_{k}(s)\right| \leq P|Q(z)-Q(s)|^{1 / 2}
$$

for all integers $k \geq 0$ and $z, s \in I_{\bar{a}}$, which implies that by Ascoli-Arzela's theorem there is a subsequence $\left\{\delta_{k(l)}(\bar{z})\right\}$ which converges uniformly to some continuous function $\delta(z)$ defined on $I_{\bar{a}}$.

Now, for $m \geq n \geq k(l+1)$, since $m-1 \geq n-1 \leq k(l)$, by using Doob's and Schwarz's inequalities and condition $1^{0}$ of $H_{2}$, we get

$$
\begin{gathered}
\delta_{m n}(z)=E\left\|u_{z}^{m}-u_{z}^{n}\right\|^{2} \\
\leq 2 E\left(\sup _{\theta \in I_{z}}\left|\int_{I_{\theta}}\left(f\left(\tau, u_{\tau}^{m-1}\right)-f\left(\tau, u_{\tau}^{n-1}\right)\right) d \tau\right|^{2}+\sup _{\theta \in I_{z}}\left|\int_{I_{\theta}}\left(g\left(\tau, u_{\tau}^{m-1}\right)-g\left(\tau, u_{\tau}^{n-1}\right)\right) d w(\tau)\right|^{2}\right) \\
\leq A \int_{I_{z}} G\left(\tau, E\left\|u_{\tau}^{m-1}-u_{\tau}^{n-1}\right\|^{2}\right) d \tau \\
\leq A \int_{I_{z}} G\left(\tau, \delta_{k(l)}(\tau)\right) d \tau
\end{gathered}
$$

for all $z \in I_{\bar{a}}$, where $A=2\left(4+a_{1} a_{2}\right)$, from which we deduce

$$
\delta_{k(l+1)}(z) \leq A \int_{I_{z}} G\left(\tau, \delta_{k(l)}(\tau)\right) d \tau, \quad z \in I_{\bar{a}} .
$$

Thus, since $\left\{\delta_{k(l)}(z)\right\}$ converges uniformly to $\delta(z)$ as $l \rightarrow \infty$, by the dominated convergence theorem of Lebesgue and the continuity of $G(z, v)$ in $v$ for each fixed $z \in I_{\bar{a}}$, we have

$$
\delta(z) \leq A \int_{I_{z}} G(\tau, \delta(\tau)) d \tau, \quad z \in I_{\bar{a}} .
$$

Therefore, by condition $2^{0}$ of $H_{2}$, we get $\delta(z) \equiv 0$ on $I_{\bar{a}}$.

Now, for $m \geq n \geq k(l+1)$, applying Doob's martingale inequality and Schwarz's inequality, we obtain

$$
\begin{gathered}
\underset{z \in I_{\bar{a}}}{E\left(\sup _{z \in I^{\prime}}\left|u^{m}(z)-u^{n}(z)\right|^{2}\right)} \\
\leq 2 E\left(\sup _{z \in I_{\bar{a}}}\left|\int_{I_{z}}\left(f\left(\tau, u_{\tau}^{m-1}\right)-f\left(\tau, u_{\tau}^{n-1}\right)\right) d \tau\right|^{2}+\sup _{z \in I_{\bar{a}}}\left|\int_{I_{z}}\left(g\left(\tau, u_{\tau}^{m-1}\right)-g\left(\tau, u_{\tau}^{n-1}\right)\right) d w(\tau)\right|^{2}\right) \\
\leq 2\left(4+a_{1} a_{2}\right) \int_{I_{\bar{a}}} G\left(\tau, E\left\|u_{\tau}^{m-1}-u_{\tau}^{n-1}\right\|^{2}\right) d \tau \\
\leq 2\left(4+a_{1} a_{2}\right) \int_{I_{\bar{a}}} G\left(\tau, \delta_{k(l)}(\tau)\right) d \tau \rightarrow 0
\end{gathered}
$$

as $l \rightarrow \infty$, which implies that the sequence $\left\{u^{n}(z)\right\}$ is a Cauchy sequence in Banach space $\mathscr{B}(\varphi)$. Therefore, there exists a stochastic process $u(z)$ such that

$$
E\left(\sup _{z \in I_{\bar{a}}}\left|u^{n}(z)-u(z)\right|^{2}\right) \rightarrow 0
$$


as $n \rightarrow \infty$. As usual, we can prove that $u(z)$ is a local solution of (1), (2).

Finally, we shall show the uniqueness of the local solution. Let $u(z)$ and $v(z)$ be two local solutions of existing on the rectangle $I_{\bar{a}}$ with $u(z)=v(z)=\varphi(z), z \in I_{0}$. Then, we get

$$
E\left\|u_{z}-v_{z}\right\|^{2} \leq 2\left(4+a_{1} a_{2}\right) \int_{I_{z}} G\left(\tau, E\left\|u_{\tau}-v_{\tau}\right\|^{2}\right) d \tau,
$$

for all $z \in I_{\bar{a}}$ from which using condition $2^{0}$ of $H_{2}$, we find $E\left\|u_{z}-v_{z}\right\|^{2}=0$ for all $z \in I_{\bar{a}}$. Therefore, we must have $u_{z}=v_{z}$ for all $z \in I_{\bar{a}}$, so $u(z)=v(z), z \in \tilde{I}$ a.s. This completes the proof.

\section{The Global Existence of Solutions}

Now, we shall present the existence and uniqueness of global solution of (1), (2).

Theorem 2: Let

$1^{0} \quad$ Assumptions $H_{1}$ and $1^{0}$ of $H_{2}$ are satisfied with $r=\infty$ and $a=\infty$ (i.e., $a_{1}=\infty$ and $\left.a_{2}=\infty\right)$;

$2^{0} \quad$ for any fixed $a>0$, the differential equation (6) has a global solution for any initial functions $\alpha$ and $\beta$;

$3^{0} \quad$ for any fixed $a>0$, condition $2^{0}$ of $\mathrm{H}_{2}$ holds.

Then, the sequence $\left\{u^{n}(z)\right\}$ defined by (5) converges uniformly on any subrectangle $I_{a}$ of $\mathbb{R}^{2}$, to a unique solution of (1), (2).

Proof: Denote by $I_{q^{*}}$ the largest rectangle on which the sequence $\left\{u^{n}(z)\right\}$ converges uni-formly. By Theorem 1 , we have that $a_{1}^{*}>0$ and $a_{2}^{*}>0$. Now, we suppose that $a_{1}^{*}<\infty$. Then, we can take $\tilde{a}_{1}$ such that $a_{1}^{*}>0$ and $a_{2}^{*}>0$. Now, we suppose that $a_{1}^{*}<\infty$. Then, we can take $\tilde{a}_{1}$ such that $a_{1}^{*}<\tilde{a}_{1}<\infty$. Thus, by assumptions $1^{0}$ and $2^{0}$, we have a solution $v(z)$ of $(6)$ with $a=\tilde{a}=\left(\tilde{a}_{1}, a_{2}\right)$ which exists on $I_{\tilde{a}}$, and estimate (8) holds on the rectangle $I_{\tilde{a}}$. The rest of the proof follows as in Theorem 1, replacing $\bar{a}$ by $\tilde{a}$. Similar arguments apply to the case $a_{2}^{*}<\infty$. This proves the theorem.

The following corollary gives a special case of the comparison function $G$.

Corollary: Assume that

$1^{0} \quad$ there are a continuous, monotone nondecreasing and concave function $\mu: \mathbb{R}_{+} \rightarrow \mathbb{R}_{+}$with $\mu(0)=0$ and $\lambda \in L_{l o c}^{1}\left(R_{+}, R_{+}\right)$such that

$$
|f(z, v)-f(z, \bar{v})|^{2}+|g(z, v)-g(z, \bar{v})|^{2} \leq \lambda(z) \mu\left(\|v-\bar{v}\|^{2}\right),
$$

for all $v, \bar{v} \in C, z \in R_{+}^{2}$;

$2^{0} \quad|f(\cdot, 0)|,|g(\cdot, 0)| \in L_{l o c}^{2}\left(R_{+}^{2}, R_{+}\right)$;

$3^{0} \quad \int_{0}^{+} 1 / \mu(\xi) d \xi=\infty$;

$4^{0} \quad$ condition $3^{0}$ of $H_{1}$ is satisfied.

Then, on any finite rectangle $I_{a}$, the sequence $\left\{u^{n}(z)\right\}, z \in I_{a}$, defined by (5), converges uniformly to a unique solution of (1), (2).

Proof: Since $\mu$ is concave by condition $1^{0}$, we obtain

$$
E|f(z, v)-f(z, \bar{v})|^{2}+E|g(z, v)-g(z, \bar{v})|^{2} \leq \lambda(z) \mu\left(E\|v-\bar{v}\|^{2}\right) .
$$


From condition $1^{0}$ we get

$$
\begin{gathered}
|f(z, v)|^{2}+|g(z, v)|^{2} \\
\leq 2\left[|f(z, v)-f(z, 0)|^{2}+|g(z, v)-g(z, 0)|\right]^{2}+2\left[|f(z, 0)|^{2}+|g(z, 0)|^{2}\right] \\
\leq 2 \lambda(z) \mu\left(\|v\|^{2}\right)+2\left[|f(z, 0)|^{2}+|g(z, 0)|^{2}\right] .
\end{gathered}
$$

Since $\mu$ is concave, there exist positive real numbers $c$ and $d$ such that $\mu(\xi) \leq c \xi+d$. Set $\varrho(z)=2 c \lambda(z)$ and $\nu(z)=2 d \lambda(z)+2|f(z, 0)|^{2}+2|g(z, 0)|^{2}$. Then $\varrho$ and $\nu$ are locally integrable with respect to $z \in R_{+}^{2}$. Hence, combining with (11) we have

$$
|f(z, v)|^{2}+|g(z, v)|^{2} \leq \varrho(z)\|v\|^{2}+\nu(z) .
$$

Next, set $H(z, v)=\varrho(z) v+\nu(z), v \geq 0$, for all $z \in R_{+}^{2}$. Then, since $H$ is linear in $v$, condition $2^{0}$ of Theorem 2 holds. Obviously, the rest of the assumptions of Theorem 2 are satisfied. Therefore, by Theorem 2, we get the desirable conclusion, which completes the proof of the corollary.

\section{Examples} (1).

We now give a few examples of differential equations which can be transformed to equation

If we define

$$
\begin{aligned}
f(x, y, v) & =\tilde{f}(x, y, v(\alpha(x, y)-x, \beta(x, y)-y)) \\
g(x, y, v) & =\tilde{g}(x, y, v(\gamma(x, y)-x, \delta(x, y)-y))
\end{aligned}
$$

then the differential equation with retarded argument

$$
\frac{\partial^{2} u}{\partial x \partial y}(x, y)=\tilde{f}(x, y, u(\alpha(t, x), \beta(x, y)))+\widetilde{g}(x, y, u(\gamma(x, y), \delta(x, y))) \frac{\partial^{2} w}{\partial x \partial y}(x, y),
$$

where $\alpha, \beta, \gamma, \delta: I_{a} \rightarrow \mathbb{R}$ are given functions such that $-h_{1} \leq \alpha(x, y) \leq x,-h_{2} \leq \gamma(x, y) \leq x$, can be transformed to equation (1).

The differential-integral equation

$$
\begin{aligned}
& \frac{\partial^{2} u}{\partial x \partial y}(x, y)=\tilde{f}\left(x, y, \int_{\alpha(x, y)}^{\tilde{\alpha}(x, y)} \int_{\beta(x, y)}^{\tilde{\beta}(x, y)} K_{1}(\xi, \tau, x, y) u(\xi, \tau) d \xi d \tau\right) \\
& +\tilde{g}\left(x, y, \int_{\gamma(x, y)}^{\tilde{\gamma}(x, y)} \int_{\delta(x, y)}^{\tilde{\delta}(x, y)} K_{2}(\xi, \tau, x, y) u(\xi, \tau) d \xi d \tau\right) \frac{\partial^{2} w}{\partial x \partial y}(x, y)
\end{aligned}
$$

can be rewritten to the particular case of (1). In this case we define $f$ and $g$ by

$$
f(x, y, v)=\tilde{f}\left(x, y, \int_{\alpha(x, y)}^{\tilde{\alpha}(x, y)} \int_{\beta(x, y)}^{\tilde{\beta}(x, y)} K_{1}(\xi, \tau, x, y) v(\xi-x, \tau-y) d \xi d \tau\right)
$$




$$
g(x, y, v)=\tilde{g}\left(x, y, \int_{\gamma(x, y)}^{\tilde{\gamma}(x, y)} \int_{\delta(x, y)}^{\tilde{\delta}(x, y)} K_{2}(\xi, \tau, x, y) v(\xi-x, \tau-y) d \xi d \tau\right)
$$

Let us define $\tilde{\varphi}$ on $\left[-\widetilde{h}_{1}, 0\right] \times\left[-\widetilde{h}_{2}, 0\right]$, where $\tilde{h}_{i} \bar{\sim}_{h_{i}}+a_{i}, i=\underset{\sim}{1}, 2$, by $\tilde{\varphi}(x, y)=\varphi(x, y)$ for $(x, y) \in I_{0}$ and $\tilde{\varphi}(x, y)=\varphi\left(-h_{1},-h_{2}\right)$ for $(x, y) \in\left[-\tilde{h}_{1},-h_{1}\right) \times\left[-\widetilde{h}_{2},-h_{2}\right)$. We introduce the operator

$$
\mathrm{g}_{(x, y)}: C\left(\left[-\tilde{h}_{1}, 0\right] \times\left[-\tilde{h}_{2}, 0\right], R\right) \rightarrow C\left(\left[x-\tilde{h}_{1}, x\right] \times\left[y-\tilde{h}_{2}, y\right], R\right),
$$

$\underset{\widetilde{h}}{\text { where }}(x, y) \in I_{a}$, which is defined by $\left(g_{(x, y)} v\right)(\xi, \tau)=v(\xi-x, \tau-y),(\xi, \tau) \in\left[x-\widetilde{h}_{1}, x\right] \times$ $\left[y-\tilde{h}_{2}, y\right]$. If we take

$$
\begin{aligned}
& f(x, y, v)=\tilde{f}\left(x, y,\left(V_{1}\left(g_{(x, y)} v\right)\right)(x, y)\right), \\
& g(x, y, v)=\tilde{g}\left(x, y,\left(V_{2}\left({ }_{(x, y)} v\right)\right)(x, y)\right),
\end{aligned}
$$

then the differential-functional equation

$$
\begin{gathered}
\frac{\partial^{2} u}{\partial x \partial y}(x, y)=\tilde{f}\left(x, y,\left(V_{1} u\right)(x, y)\right)+\tilde{g}\left(x, y,\left(V_{2} u\right)(x, y)\right) \frac{\partial^{2} w}{\partial x \partial y}(x, y) \quad(x, y) \in I_{a} \\
u(x, y)=\tilde{\varphi}(x, y),(x, y) \in\left[-\tilde{h}_{1}, 0\right] \times\left[-\widetilde{h}_{2}, 0\right]
\end{gathered}
$$

where $V_{j}, j=1,2$, are operators of the Volterra type, can be transformed to the particular case of (1), (2).

\section{References}

[1] Cairoli, R. and Walsh, J.B., Stochastic integrals in the plane, Acta Math. 134 (1975), 111183.

[2] Gikhman, I.I. and Pjasetskaja, T.E., On existence of solutions for a class of partial stochastic equations, Dopovidi Akad. Nauk. Ukraini 5 (1977), 387-390 (in Russian).

[3] Kisielewicz, M., Some Properties of Partial Functional Differential Equations of the Hyperbolic Type, Lubuskie Towarzystwo Naukowe, Poznań - Zielona Góra 1971 (in Polish).

[4] Pelczar, A., Some functional differential equations, Dissertationes Math 100 (1973), 3-100.

[5] Rodkina, A.E., On the existence and uniqueness of solutions of stochastic differential equation with hereditary, Stochastics 12 (1984), 187-200.

[6] Taniguchi, T., Successive approximations to solutions of stochastic differential equations, J. Diff. Eq. 96 (1992), 152-169.

[7] Tsarenko, T.I., On some scheme of the construction of stochastic integral for the random field, Kibernetika 1 (1972), 113-118 (in Russian).

[8] Tsarenko, T.I., On existence and uniqueness of solutions of stochastic Darboux equation, Kibernetika 4 (1972), 115-117 (in Russian). 


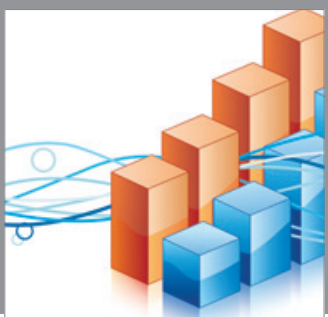

Advances in

Operations Research

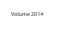

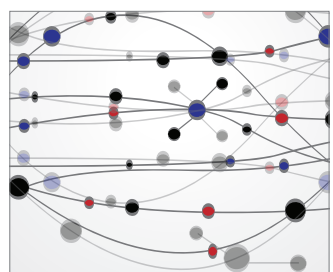

\section{The Scientific} World Journal
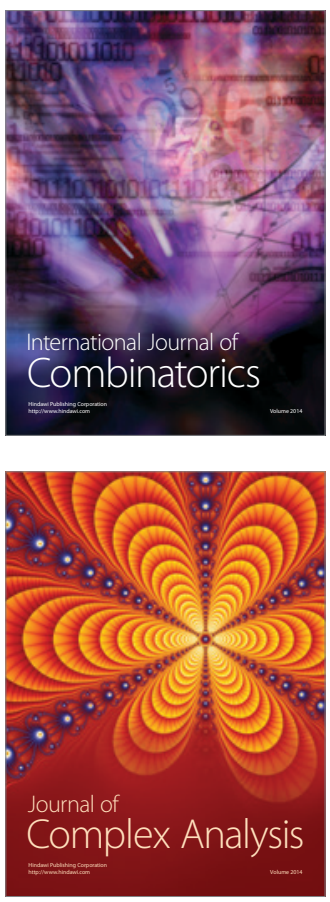

International Journal of

Mathematics and

Mathematical

Sciences
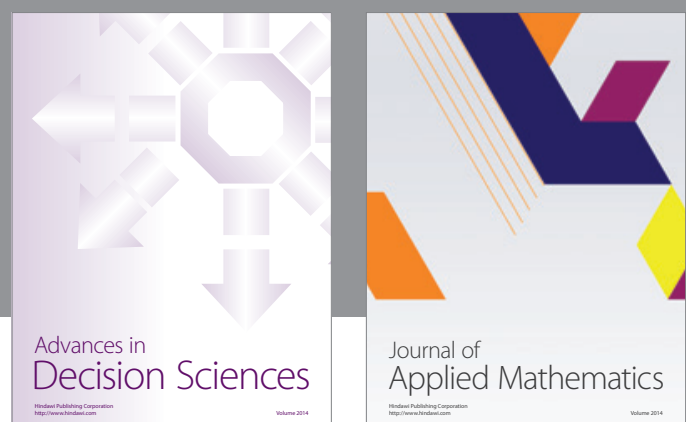

Journal of

Applied Mathematics
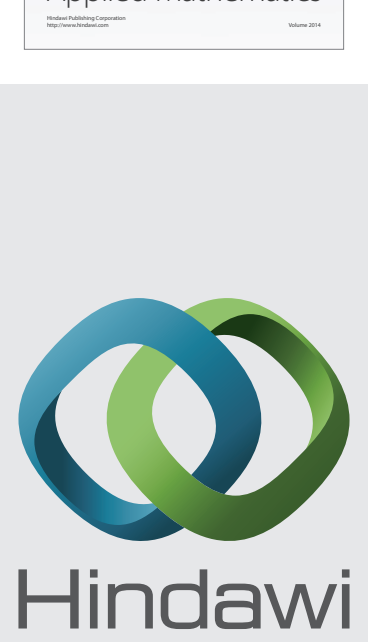

Submit your manuscripts at http://www.hindawi.com
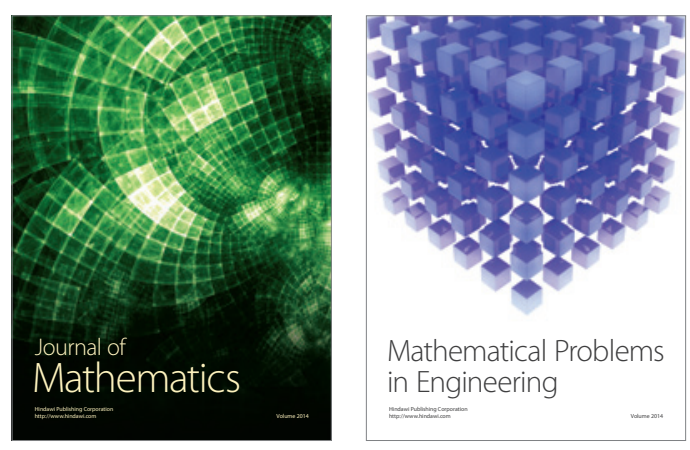

Mathematical Problems in Engineering
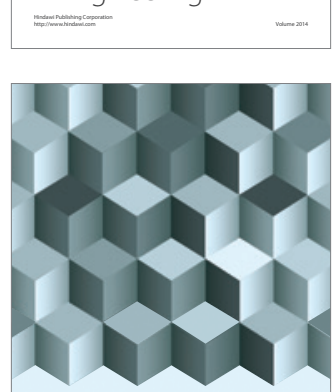

Journal of

Function Spaces
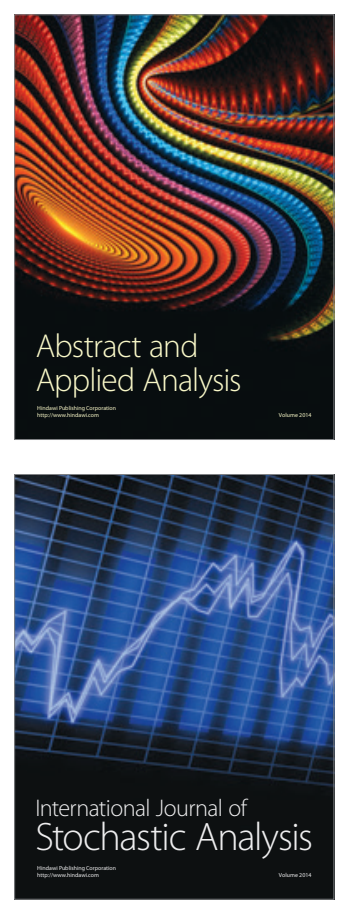

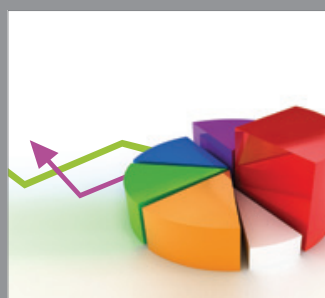

ournal of

Probability and Statistics

Promensencen
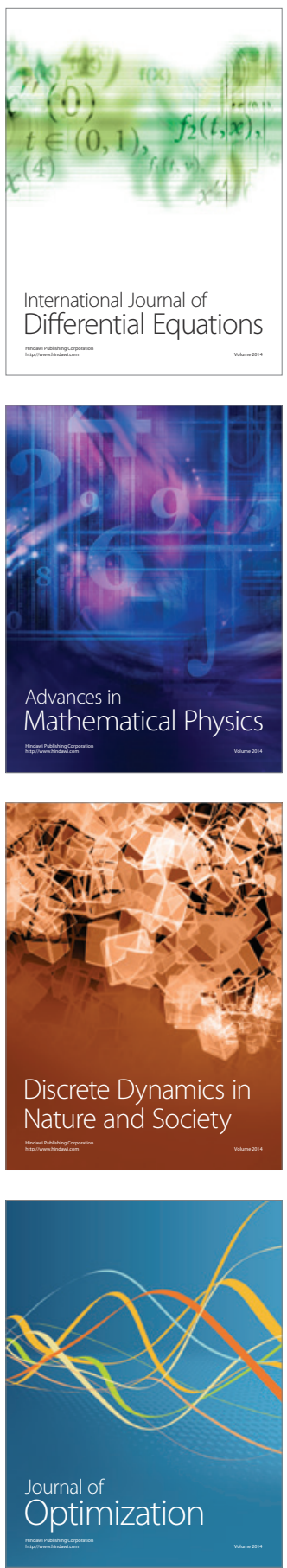